\title{
The evolving point of references
}

\author{
The use of a bibliography in scientific literature has evolved over time. Here, we look at its history and the roles it \\ plays in contemporary science.
}

\section{T} he Philosophical Transaction of the Royal Society is considered by many historians as the first modern scientific journal. Its first issue, published in 1665, contains several articles that feature many characteristic elements of contemporary scientific literature, including some references. The practice of including a bibliography section into scientific articles, however, was not yet very systematic in the seventeenth century. In fact, referencing the work of peers in a formal manner became more common throughout the nineteenth century, although the lack of an exhaustive bibliography was not considered as a reason to prevent publication of scientific works - the first edition of Origin of Species by Charles Darwin is a good example ${ }^{1}$. One would have to wait until the twentieth century to witness the introduction of stringent requirements for citations and bibliography sections as regular components of scientific literature. When Taylor published his theory of catalytic surfaces in 1925, for instance, the article already contained ten citations, including a reference to unpublished results ${ }^{2}$.

In the middle of the twentieth century, the interest in references as descriptors for the scientific endeavour became so widespread that people like Eugene Garfield - the father of what later became the Web of Science - and Derek de Solla Price developed approaches for the systematic analysis of the body of literature produced by scientists in different areas, establishing the field of scientometrics at the same time. The merit of this approach has been to reveal the structure of modern scientific literature, which is configured in the form of a network of scientific papers ${ }^{3}$ interlinked by means of citations.

This brief historical excursion on the use of references may come as a surprise to some researchers; nowadays, however, authors of scientific articles are very much aware of the importance of including a well-thought-out reference list in their manuscripts. In fact, the selected literature is essential to define the context and background of the study. Claims from previous articles are often used as a rationale to justify the goal of a new manuscript, especially when the performances of known processes are being improved or when facts are challenged - for example, challenge to someone's interpretation of a given catalytic mechanism. Eventually, precedents from the literature are also used to back up the choice of specific control experiments and to comply with best practice in a certain field ${ }^{4}$.

\section{"Authors of scientific articles are very much aware of the importance of including a well-thought-out reference list in their manuscripts."}

While performing the initial assessment of a manuscript under consideration at Nature Catalysis, we evaluate the selected reference list as an integral part of the work. Usually, the handling editor performs an independent literature search in order to establish the significance of the study and evaluate if any relevant precedents have not been considered. Occasionally, a manuscript may fail to pass the initial editorial assessment if related previous work, which compromises the level of advance of the study, has not been covered. If an article is sent out for peer review, we invite the reviewers to comment on the extent to which the claims of the paper represent a significant advance in the field. In this regard, we ask them to provide relevant references where possible, in case the conclusions are not original.

Despite their importance, references do not come without complications and may represent a weak spot for potential forms of misconduct. The Committee on Publication Ethics (COPE), for instance, has recently highlighted a series of problems relating to citation manipulation. One of these issues is the use of excessive self-citation. While there may be good reasons for authors to refer to their previous work - an article could be the genuine continuation of a previous study - self-citation may actually reflect an attempt to artificially inflate citation rates. Recent bibliometric studies have renewed attention to this problem ${ }^{5}$ but, while this habit should not be encouraged, we are still missing a consensus on what objective metrics should be used to establish acceptable levels of self-citation. Obviously, the root of the problem must be found in the known practice of overly relying on citation-based metrics to award tenures and evaluate career progress in academia. This represents a questionable development of the function of bibliographies and has long been discouraged by several funding agencies, academic institutions and publishers including Springer Nature.

Yet, the problem of self-citation does not only concern authors - a very similar rationale may lead peer reviewers to request citation of their previous publications. Reviewers are generally selected by the editors based on their experience in a specific area of research. As such, they have often contributed to the field and a reference to a given article may benefit the overall message of the manuscript. However, in some cases, suggestions to include multiple references to the work of a specific research group are made, which may be redundant. It is ultimately the editor's responsibility to verify the relevance of the suggested literature and intervene where necessary. The list of selected references should always be balanced and concise.

Overall, there is a sort of qualifying function that can be attributed to the references within an article, and we would like to recall it here with the words of philosopher Bruno Latour: "A document becomes scientific when its claims stop being isolated and when the number of people engaged in publishing it are many and explicitly indicated in the text" ${ }^{\prime}$. In other words, references remind investigators that science is a community enterprise.

Published online: 14 December 2020 https://doi.org/10.1038/s41929-020-00562-1

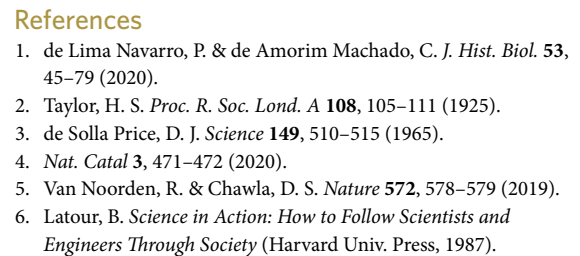

